Cite this: Nanoscale, 2013, 5, 8656

\title{
Protein corona affects the relaxivity and MRI contrast efficiency of magnetic nanoparticles $\dagger$
}

\author{
Houshang Amiri, ${ }^{\text {*a }}$ Lorenzo Bordonali, ${ }^{e}$ Alessandro Lascialfari, ${ }^{\text {ef }}$ Sha Wan, ${ }^{\mathrm{b}}$ \\ Marco P. Monopoli, ${ }^{b}$ Iseult Lynch,,$^{* b}$ Sophie Laurent ${ }^{\mathrm{g}}$ and Morteza Mahmoudi ${ }^{\star c d}$
}

Magnetic nanoparticles (NPs) are increasingly being considered for use in biomedical applications such as biosensors, imaging contrast agents and drug delivery vehicles. In a biological fluid, proteins associate in a preferential manner with NPs. The small sizes and high curvature angles of NPs influence the types and amounts of proteins present on their surfaces. This differential display of proteins bound to the surface of NPs can influence the tissue distribution, cellular uptake and biological effects of NPs. To date, the effects of adsorption of a protein corona (PC) on the magnetic properties of NPs have not been considered, despite the fact that some of their potential applications require their use in human blood. Here, to investigate the effects of a PC (using fetal bovine serum) on the MRI contrast efficiency of superparamagnetic iron oxide NPs (SPIONs), we have synthesized two series of SPIONs with variation in the thickness and functional groups (i.e. surface charges) of the dextran surface coating. We have observed that different physico-chemical characteristics of the dextran coatings on the SPIONs lead to the formation of PCs of different compositions. ${ }^{1} \mathrm{H}$ relaxometry was used to obtain the longitudinal, $r_{1}$, and transverse, $r_{2}$, relaxivities of the SPIONs without and with a PC, as a function of the Larmor frequency. The transverse relaxivity, which determines the efficiency of negative contrast agents (CAs), is very much dependent on the functional group and the surface charge of the SPIONs' coating. The presence of the PC did not alter the relaxivity of plain SPIONs, while it slightly increased the relaxivity of the negatively charged SPIONs and dramatically decreased the relaxivity of the positively charged ones, which was coupled with particle agglomeration in the presence of the proteins. To confirm the effect of the PC on the MRI contrast efficiency, in vitro MRI experiments at $\nu=8.5 \mathrm{MHz}$ were performed using a low-field MRI scanner. The MRI contrasts, produced by different samples, were fully in agreement with the relaxometry findings.

Received 20th January 2013

Accepted 18th June 2013

DOI: $10.1039 / c 3 n r 00345 k$

www.rsc.org/nanoscale formation of a protein "corona" that is strongly associated with the NPs' surface and that defines how living organisms (e.g. cells) "see" the NPs in a biological milieu. ${ }^{1-5}$ According to previous reports, the cell "sees" a nano-system in which the NPs are covered by a "hard" corona of slowly exchanging proteins with a surrounding "soft" corona consisting of weakly interacting and rapidly exchanging proteins. ${ }^{6}$ The composition of the protein corona (PC) is highly dependent on the physicochemical properties of the NPs such as their size, composition, and surface characteristics. ${ }^{7}$

Superparamagnetic iron oxide nanoparticles (SPIONs) have been recognized as very promising nanosystems with good biocompatibility ${ }^{8,9}$ and high potential to be used for diagnosis and therapy simultaneously., ${ }^{\mathbf{8} 10-15}$ SPIONs have been extensively studied for both in vitro and in vivo biomedical applications, such as contrast enhancement for magnetic resonance imaging (MRI) ${ }^{8,16-21}$ tissue specific release of therapeutic agents, ${ }^{22}$ hyperthermia, ${ }^{23}$ transfection, ${ }^{23}$ cell/biomolecule separation, ${ }^{23}$ and targeted drug delivery. ${ }^{22}$ While these applications rely on the magnetic properties of the SPIONs, some materials that have 
shown excellent magnetic properties have run into severe validation difficulties for use in clinical applications. One of the main features that can potentially cause changes in the in vivo magnetic properties of SPIONs is their possible interactions with the surrounding proteins or other biomolecules in the blood. Therefore, probing the interactions of SPIONs with plasma proteins, in order to understand the composition of the adsorbed protein layer and its effect on SPION magnetic functionality, is essential. $^{5}$

SPIONs are known as negative (or $T_{2}$-weighted) MRI contrast agents (CAs), which decrease the signal intensity of the hydrogen nuclei within the tissues/regions where they are delivered. ${ }^{24}$ The main advantage of SPIONs as MRI CAs is their high sensitivity, resulting in significant contrast enhancement in MR images. ${ }^{25}$ There has been great interest in synthesizing novel CAs and studying the influence of microstructural parameters such as the magnetic core size on the MRI contrast efficiency. To our knowledge, the effect of the formation of a PC on the efficiency of SPIONs as MRI CAs has not been reported.

In this article we synthesize two different groups of SPIONs: (i) single (S-series) and (ii) double (D-series) thicknesses of dextran coating, each group consisting of different surface chemistries/charges in the dextran coating (i.e. plain, negative and positive surface charges). We study the physico-chemical and magnetic properties, and the MRI contrast efficiency of the aforementioned SPIONs in the absence and presence of a PC.

\section{Materials and methods}

Analytical grade iron salts (i.e. iron chloride) and sodium hydroxide $(\mathrm{NaOH})$ were purchased from Merck Inc. and used without further purification. Dextran with an average molecular weight of 5000, dimethylsulfoxide, sodium periodate, potassium cyanide, and ammonium persulfate were purchased from Sigma-Aldrich and used as received.

\section{Preparation of the carboxyl-dextran}

The carboxylated dextran was prepared according to a procedure reported elsewhere. ${ }^{26}$ Initially, the hydroxyl groups in the dextran were oxidized to aldehyde groups by sodium periodate. ${ }^{27}$ Briefly, sodium periodate was dissolved in de-oxygenated de-ionized (DI) water and introduced to a dextran solution (4 g in $30 \mathrm{~mL}$ of de-oxygenated DI water). The resulting solution was homogenized for 2 hours at room temperature followed by dialysis against water in a membrane bag with a 1000 molecular weight $\left(M_{\mathrm{w}}\right)$ cut-off for 4 days. The resulting solution was reacted with potassium cyanide to prepare a cyanohydrin intermediate. Finally, carboxylic acid groups were created on the terminal units of dextran by hydrolysis of the obtained cyanohydrin intermediate. The prepared carboxylated dextran was lyophilized and stored at $-80{ }^{\circ} \mathrm{C}$.

\section{Preparation of the amino-dextran}

The amino dextran was prepared according to a procedure reported elsewhere. ${ }^{28}$ Briefly, $10 \mathrm{~g}$ of dextran was dissolved in $75 \mathrm{~mL}$ of de-oxygenated DI water containing $2.5 \mathrm{~g}$ sodium hydroxide and $0.2 \mathrm{~g}$ sodium borohydride at $\mathrm{pH} 11$, which was fixed by dropwise addition of $2.5 \mathrm{~N} \mathrm{NaOH}$ and $2 \mathrm{~mL}$ allyl bromide, at a temperature of around $50{ }^{\circ} \mathrm{C}$. Acetic acid was used to neutralize the solution, followed by incubation of the solution at $4{ }^{\circ} \mathrm{C}$ for 2 hours. The top organic layer was removed and $100 \mathrm{~mL}$ of fresh de-oxygenated DI water was added and the solution was dialyzed using a membrane bag with a $50000 M_{\mathrm{w}}$ cut-off for $24 \mathrm{~h}$. In order to prepare the amino dextran conjugate, the dialyzed mixture was reacted with $7.5 \mathrm{~g}$ of aminoalkyl thiol in $30 \mathrm{~mL}$ dimethylsulfoxide, with $0.1 \mathrm{~g}$ ammonium persulfate used as the initiator. After 3 hours, the same volume of fresh de-oxygenated DI water was added to the reactor, followed by $\mathrm{pH}$ adjustment using sodium hydroxide, and the product was diluted with $140 \mathrm{~mL}$ sodium acetate buffer $\left(0.02 \mathrm{~mol} \mathrm{~L}^{-1}, \mathrm{pH} 4\right)$. In order to ensure the removal of excess materials, the obtained amino dextran conjugate was dialyzed using a membrane bag with a $50000 M_{\mathrm{w}}$ cut-off for $24 \mathrm{~h}$. The prepared amino dextran conjugate was lyophilized and stored at $-80{ }^{\circ} \mathrm{C}$.

\section{Synthesis of ultra-small SPIONs with various surface characteristics}

In order to ensure the deoxygenation of DI water, all solutions were bubbled with neutral gas (i.e. argon) for 30 minutes. The iron salts (i.e. $\mathrm{FeCl}_{3}$ and $\mathrm{FeCl}_{2}$ ) were dissolved in separate beakers containing $1.5 \mathrm{M} \mathrm{HCl}$ prepared using de-oxygenated DI water. The obtained iron salt solutions were blended together by adjusting to a molar fraction of $2\left(\mathrm{Fe}^{3+} / \mathrm{Fe}^{2+}\right)$. Various dextrans, including plain-, carboxylated-, and amino conjugated-dextrans were dissolved in de-oxygenated DI water. The three types of dextrans were individually mixed with the iron salt solutions and introduced into a three-neck flask equipped with a homogenizer, with stirring at $10000 \mathrm{rpm}$. This procedure was repeated three times to obtain SPIONs with various surface coatings (i.e. negative (carboxylated), plain, and positive (amine conjugate)). In order to obtain the single coated NPs, the dextran/iron mass ratio was fixed at $2 .^{29}$

The SPIONs were formed by dropwise addition of a base medium (i.e. $\mathrm{NaOH}$ ) into the prepared dextran and iron salt mixtures with vigorous stirring under argon protection. In order to achieve highly monodisperse SPIONs (i.e. to decrease the mass transfer phenomena, which may allow NPs to combine and build up larger polycrystalline particles), the reactor was transferred to an ultrasonic bath (100 Watt) in order to create a turbulent flow. ${ }^{30}$ After one hour, the black suspensions were placed in a strong magnetic field gradient produced by a permanent $\mathrm{Nd}-\mathrm{Fe}-\mathrm{B}$ magnet (with cylindrical shape, diameter $4 \mathrm{~cm}$ and height $3 \mathrm{~cm}$ exhibiting superior magnetic properties), and the dextran coated SPIONs were collected. The supernatant was completely removed and the coated SPIONs were re-dispersed in DI water several times. In order to ensure that all the excess ammonia, iron cations and free dextran macromolecules were removed, the obtained ferrofluids were dialyzed using a membrane bag with a 50000 molecular weight $\left(M_{\mathrm{w}}\right)$ cut-off for $24 \mathrm{~h}$. The obtained ferrofluids were kept at $4{ }^{\circ} \mathrm{C}$ for the protein-interaction experiments. Bare SPIONs can also be produced by employing the same procedure without using dextran. The prepared SPIONs with 
their various surface chemistries (dextran coatings) are shown schematically in Fig. 1.

\section{Preparation of double-coated SPIONs with various surface characteristics}

The three types of dextrans were each dissolved in DI water, with the same concentration as that used for the formation of the first layer (previous section), and the respective single-dextran coated SPIONs were added to the reactor containing the same charged dextran under vigorous agitation. After one hour, the double coated SPIONs were collected using a strong magnet. The supernatant was completely removed and the double coated SPIONs were re-dispersed in DI water several times. Note that for the plain-dextran coated SPIONs the characterisation data suggest that the second layer did not form successfully. In order to ensure the removal of excess ammonia, dextran macromolecules and iron, the obtained ferrofluids were dialyzed using a membrane bag with a $50000 M_{\mathrm{w}}$ cut-off for $24 \mathrm{~h}$. The obtained ferrofluids were kept at $4{ }^{\circ} \mathrm{C}$ for protein-interaction experiments.

\section{TEM, DCS, and DLS}

The physico-chemical properties of the various SPIONs were characterized as follows. The morphologies of the various SPIONs were analyzed by Transmission Electron Microscopy (TEM) operating at $200 \mathrm{kV}$. To prepare samples for TEM, a drop of the SPION suspension was placed on a copper grid and dried. Differential centrifugal sedimentation (DCS) experiments were performed with a CPS Disc Centrifuge DC24000 (see full details in the ESI $\dagger$ ). Dynamic light scattering (DLS) measurements were performed with a Malvern PCS-4700 instrument equipped with a 256-channel correlator. The $488.0 \mathrm{~nm}$ line of a Coherent Innova-70 Ar ion laser was used as the incident beam with a power of $250 \mathrm{~mW}$. The employed scattering angles, $\theta$, ranged between $40^{\circ}$ and $140^{\circ}$. The temperature was maintained at $25^{\circ} \mathrm{C}$ with an external circulator. Zeta potential determination was performed using a Malvern Zetasizer $3000 \mathrm{HSa}$.

\section{Interaction of SPIONs with proteins}

The interactions of the SPIONs were assessed using fetal bovine serum (FBS). $100 \mu \mathrm{L}$ of the ferrofluid (with a SPION concentration of $4 \mathrm{mg} \mathrm{mL}^{-1}$ ) was mixed with $900 \mu \mathrm{L}$ of $10 \% \mathrm{v} / \mathrm{v}$ FBS. The $\mathrm{PC}$ has been reported to be formed in a relatively stable manner over a period of one hour. ${ }^{3}$ Therefore, we incubated our samples for $1 \mathrm{~h}$ at $37^{\circ} \mathrm{C}$ (i.e. the normal human body temperature).

\section{Magnetic properties, relaxivity, and MRI contrast efficiency}

Magnetic properties of the SPIONs were investigated using an MPMS-XL superconductor quantum interface device (SQUID) magnetometer from Quantum Design and the experiments were performed using static magnetic fields. Magnetization of the various dextran-coated SPIONs was measured as a function of both temperature (ZFC/FC) and applied magnetic field (the socalled hysteresis). The ZFC/FC experiments were performed at a relatively low magnetic field, i.e. 0.005 T. To study the system behavior, and specifically the saturation magnetization below the blocking temperature, the hysteresis curves were acquired at $5 \mathrm{~K}$ for both S- (single-coated) and (double-coated) D-plain samples.

The MRI contrast efficiency of the samples was assessed at room temperature by investigating the hydrogen relaxivity (the relaxation rates per iron concentration, expressed in $\mathrm{mM}) .{ }^{1} \mathrm{H}$ relaxometry was used to measure longitudinal, $T_{1}$, and transverse, $T_{2}$, relaxation times over a wide range of frequencies including 8.5, 21, and $63 \mathrm{MHz}$ representative of clinical MRI systems. For frequencies in the range of $10 \mathrm{kHz}<f<10 \mathrm{MHz}$,

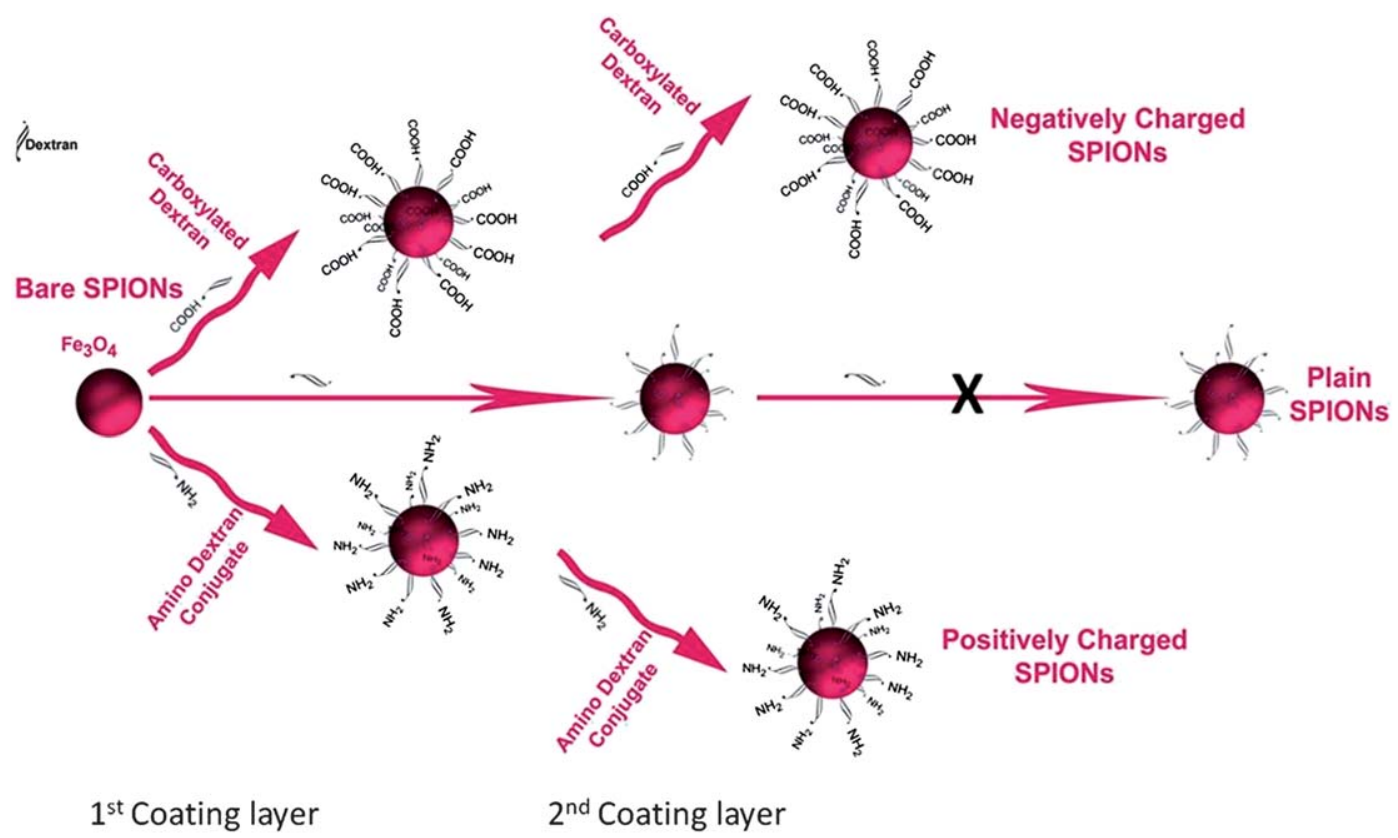

Fig. 1 Scheme of the formation of different dextran-coated SPIONs (X corresponds to the low yield process). 
data were collected with a Smartracer Stelar relaxometer using the Fast-Field-Cycling technique, while for frequencies $f>10$ $\mathrm{MHz}$, Stelar Spinmaster and Apollo-Tecmag spectrometers were used. To obtain $T_{1}$ and $T_{2}$ values, standard radio-frequency excitation sequences i.e. saturation recovery and CPMG-like were applied, respectively.

MRI experiments were performed at 8.5 $\mathrm{MHz}$ employing an Artoscan scanner from Esaote S.p.A. The images were obtained by scanning the vials containing SPION samples and Endorem ${ }^{\circledR}$ (plain dextran coated SPIONs which used to be a commercial contrast agent), before and after formation of the PC. High resolution spin echo sequences were used with the following image parameters: TR/TE/NEX $=3 \mathrm{~s} / 18 \mathrm{~ms} / 1, \mathrm{FOV}=180 \times 180$, matrix $=256 \times 192$, where TR is the repetition time, TE is the echo time, NEX is the number of averages, and FOV is the field of view.

\section{Results and discussion}

Fig. 2 and 3 show transmission electron microscopy (TEM) images and dynamic light scattering (DLS) curves of single- and double-dextran coated SPIONs. As can be seen, the SPION cores were synthesized with a narrow size distribution in the presence of the dextran layer(s) (the PDI values were all less than 0.25 in DI water, as indicated in Table 1). The SPION sizes, size distributions and surface charge were also determined. The results of DLS and zeta potential experiments are summarized in Table 1

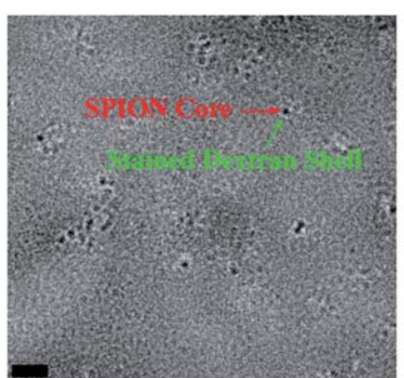

(a)

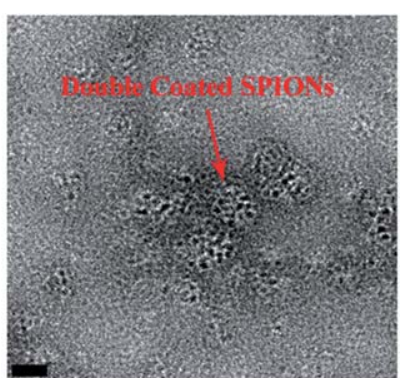

(b)
Fig. 2 TEM images of (a) single- and (b) double-coated SPIONs, respectively (the scale bar is $20 \mathrm{~nm}$ ).

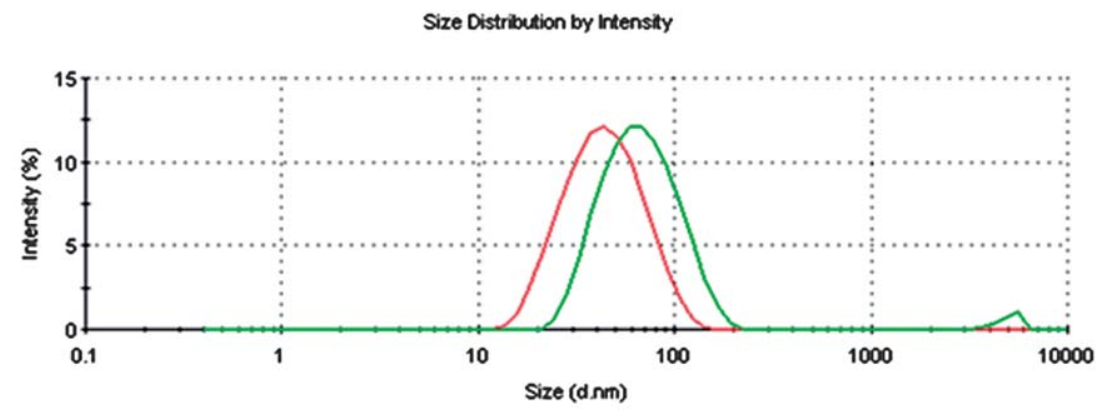

(a)

Size Distribution by intensity

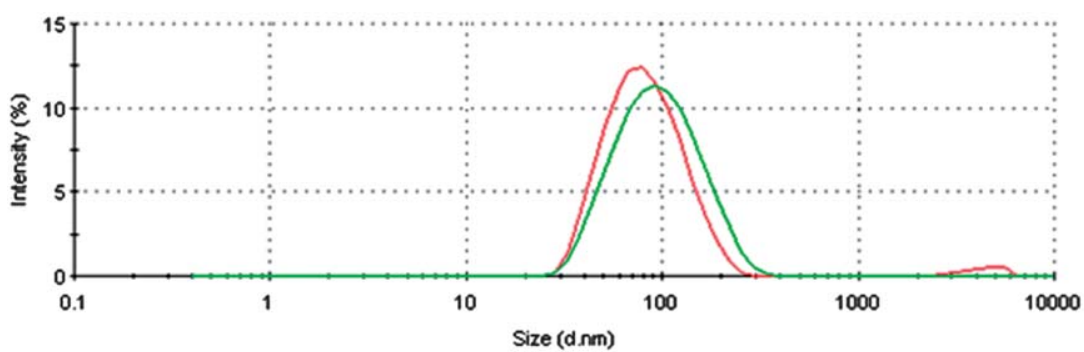

(b)

Size Distribution by Intensity

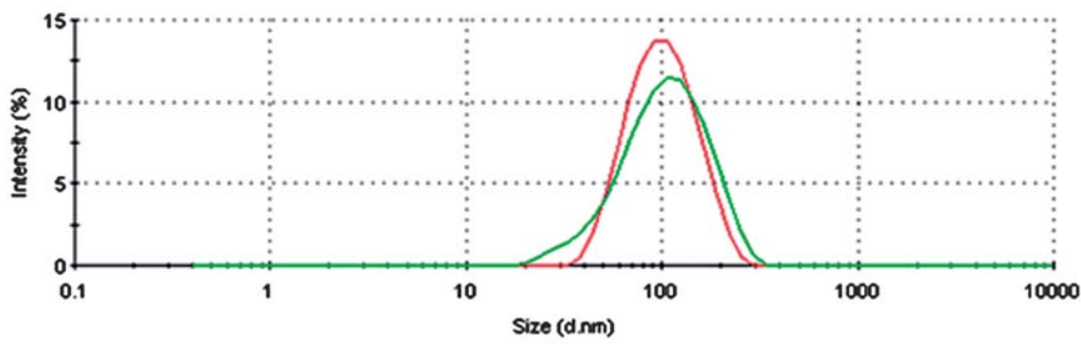

(c)

Fig. 3 DLS curves of the single coated SPIONs (red curve) and double coated SPIONs (green curve) for (a) negative, (b) plain, and (c) positive SPIONs dispersed in PBS. 
Table 1 Physico-chemical description of the various SPIONs in DI water

\begin{tabular}{|c|c|c|c|c|c|c|c|}
\hline Sample & Functional group & TEM size (nm) & $D_{\mathrm{H}}^{a}(\mathrm{~nm})$ & $\mathrm{PDI}^{b}$ & $\left\langle D_{\mathrm{H}}\right\rangle^{c}(\mathrm{~nm})$ & Zeta-potential (mV) & Nick-name \\
\hline $\begin{array}{l}\text { Bare SPION } \\
\text { (no coating) }\end{array}$ & $\mathrm{OH}$ & $5 \pm 0.5$ & - & - & - & - & Bare \\
\hline \multirow[t]{2}{*}{ coated SPION } & Plain & & $74.0 \pm 1.0$ & 0.209 & $87.6 \pm 4.5$ & $-9.1 \pm 0.7$ & S-plain \\
\hline & $\mathrm{NH}_{2}$ & & $105.1 \pm 6.2$ & 0.248 & $92.0 \pm 2.5$ & $+19.3 \pm 0.5$ & S-positive \\
\hline $\begin{array}{l}\text { Double dextran } \\
\text { coated SPION }\end{array}$ & $\mathrm{COOH}$ & $d$ & $64.6 \pm 1.3$ & 0.24 & $74.2 \pm 4.0$ & $-17.8 \pm 0.3$ & D-negative \\
\hline
\end{tabular}

${ }^{a} z$-average hydrodynamic diameter extracted by cumulant analysis of the data. ${ }^{b}$ Polydispersity index. ${ }^{c}$ Average hydrodynamic diameter determined from the CONTIN size distribution (see Fig. S1 of the ESI for the curves). ${ }^{d}$ Difficult to determine since the dextran layer is weakly scattering and there might be some agglomeration of particles during drying.

for all samples. The average TEM diameter of single-coated particles is $\sim 15 \mathrm{~nm}$ (with the iron oxide core being $\sim 5 \mathrm{~nm}$ ), however, the DLS results are highly dependent on the surface chemistry of the SPIONs' dextran coating. More specifically, the minimum hydrodynamic size is obtained for the negative particles while the maximum hydrodynamic size was obtained for the positive ones, suggesting different degrees of swelling/ hydration of the dextran layers. The addition of the second layer of dextran increased the DLS hydrodynamic diameters for the negatively and positively charged SPIONs. In the case of the plain SPIONs, it appeared that a second dextran layer did not form as no change in the particle size was observed. This suggests that the immobilized number of ions and the water layer on the surface of SPIONs are highly dependent on the surface charge and size of the NPs, and this should be considered in the description of a protein absorption phenomenon.

The NPs-hard corona complexes have been characterized by DCS to evaluate possible aggolomeration, which is partially occurring for plain and positively charged NPs and protein corona composition by SDS-PAGE. As already previously shown with the DCS measurements it is possible to correlate the protein corona shell thickness with the apparent size shift of the NPs-hard corona complexes comparing to the pristine NPs (see Table 2). The results showed that there were very significantly lower amounts of proteins in the hard coronas of the positively charged SPIONs relative to the plain and especially the negatively charged SPIONs, as shown in Fig. 4 and summarized in Table 2 (and also Fig. S2 and S3 of the ESI $\dagger$ ). The bare SPIONs

Table 2 Protein corona thickness for S- and D-series SPIONs with different dextran coating charges; corona thicknesses were calculated from the DCS data according to the core-shell method. ${ }^{3}$ (Hard corona refers to the corona following removal of unbound proteins and washing; in situ corona refers to the corona in the presence of loosely bound and unbound plasma proteins)

\begin{tabular}{lll}
\hline Sample & $\begin{array}{l}\text { Hard corona } \\
\text { thickness }(\mathrm{nm})\end{array}$ & $\begin{array}{l}\text { In situ } \\
\text { thickness }(\mathrm{nm})\end{array}$ \\
\hline S-negative & 0.69 & 3.23 \\
S-plain & 1.385 & 3.02 \\
D-negative & 1.59 & 3.99 \\
D-plain & 3.165 & 5.29
\end{tabular}

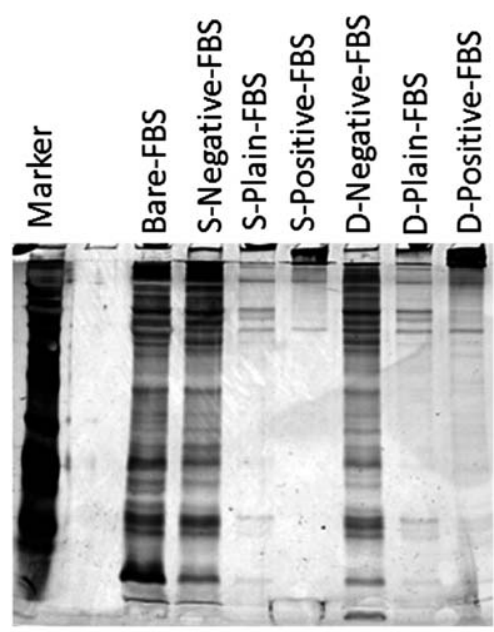

(a)

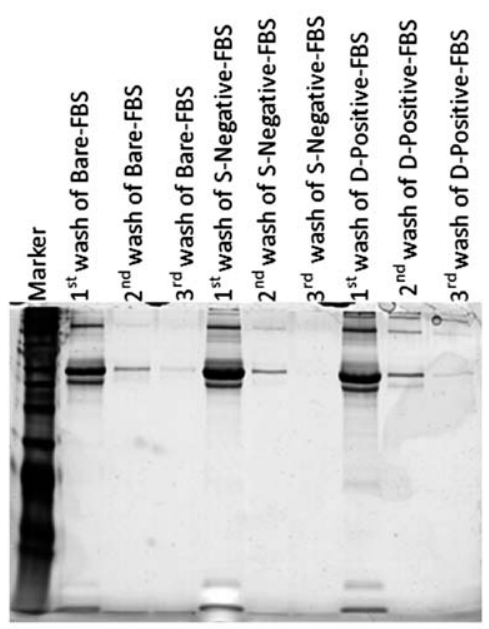

(b)

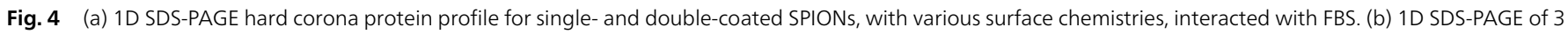
washes for bare, S-negative and D-positive. 

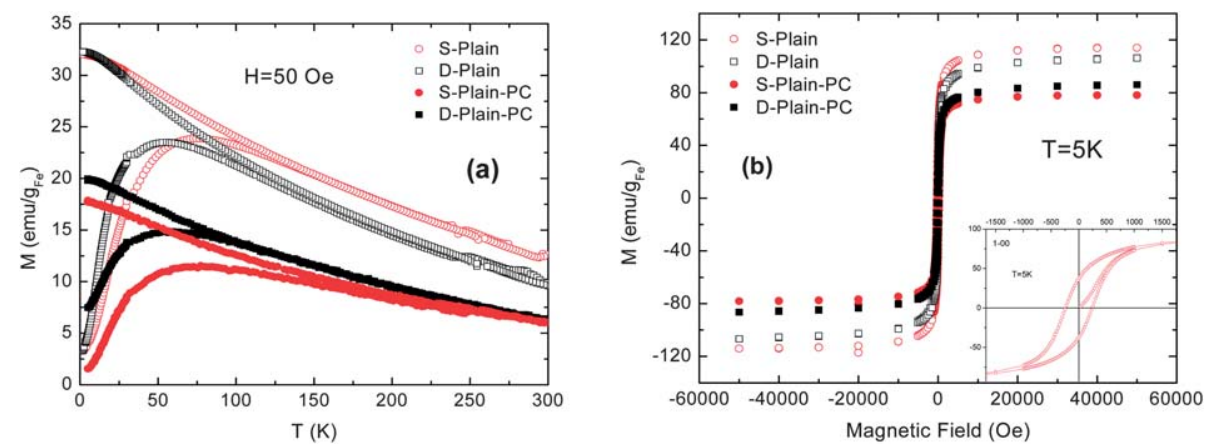

Fig. 5 Magnetization per gram of iron oxide for the S- and D-plain SPIONs as a function of (a) temperature and (b) applied magnetic field. The inset in (b) shows a zoomed view of S-plain SPIONs with the same axis units.

bound significantly more proteins than any of the dextrancoated SPIONs as expected.

The magnetization of the various SPIONs was measured as a function of temperature and applied magnetic field. Fig. 5a illustrates the magnetization versus temperature (the so-called zero-field-cooling and field cooling, ZFC/FC) experiments, used to assess the magnetization of the differently coated SPIONs, performed on S- and D-plain samples, before and after incubation with the FBS proteins, at 50 Oe. As shown in Table 3, the blocking temperature $T_{\mathrm{B}}$, the temperature corresponding to the peak in the ZFC curve below which the spins are frozen, was lower for the D-plain SPIONs compared to the S-plain ones. In the presence of the protein corona, relative to the value in the absence of proteins, $T_{\mathrm{B}}$ does not significantly change for both D- and S-plain samples.

The hysteresis curves, at $5 \mathrm{~K}$, for both S- and D-plain samples are given in Fig. 5b. The magnetization versus applied field increases with the magnetic field and saturates at a certain field, which is the typical behavior for superparamagnetic systems. The derived parameters for the SPIONs and SPION-PC complexes are presented in Table 3. According to the experimental results, remanence magnetization, $M_{\mathrm{r}}$, decreased in the presence of the PC. Both S- and D-series presented similar saturation magnetization, $M_{\mathrm{s}}$, values which were expected as they all have the same magnetic core size. The $M_{\mathrm{s}}$ values decreased when samples were associated with the PC.

To evaluate the effect of the PC on the MRI contrast efficiency of the SPIONs, the nuclear longitudinal and transverse

Table 3 Blocking temperature, $T_{\mathrm{B}}$, remanence magnetization, $M_{\mathrm{r}}$, coercive field, $H_{\mathrm{c}}$ and saturation magnetization, $M_{\mathrm{s}}$ of $\mathrm{S}$ - and D-plain samples as obtained from SQUID experiments. $r_{a}$ reports the value of the minimum approach distance as estimated from the best fit of the relaxometry profiles (i.e. Fig. 6)

\begin{tabular}{|c|c|c|c|c|c|}
\hline Sample & $T_{\mathrm{B}}(\mathrm{K})$ & $\begin{array}{l}M_{\mathrm{r}} \\
\text { (emu per } \\
\left.\mathrm{g}_{\mathrm{Fe} 3 \mathrm{O} 4}\right)\end{array}$ & $\begin{array}{l}H_{\mathrm{c}} \\
(\mathrm{Oe})\end{array}$ & $\begin{array}{l}M_{\mathrm{s}} \\
\text { (emu per } \\
\left.\mathrm{g}_{\mathrm{Fe} 3 \mathrm{O} 4}\right)\end{array}$ & $r_{\mathrm{a}}(\mathrm{nm})$ \\
\hline S-plain & 75.2 & 41 & 231 & 112 & $8.9 \pm 0.3$ \\
\hline S-plain-PC & 76.72 & 37.42 & 227.64 & 86 & $8.8 \pm 0.2$ \\
\hline D-plain & 57.22 & 37.3 & 230 & 106 & $8.2 \pm 0.2$ \\
\hline D-plain-PC & 58.05 & 30.5 & 236 & 80 & $7.7 \pm 0.2$ \\
\hline
\end{tabular}

relaxivities ( $r_{1}$ and $r_{2}$, respectively) were obtained from the spinlattice, $T_{1}$, and the spin-spin, $T_{2}$, relaxation times, measured at room temperature as a function of Larmor frequency, as: $:^{23,31}$

$$
r_{i}=\left[\left(1 / T_{i}\right)_{\mathrm{s}}-\left(1 / T_{i}\right)_{\mathrm{d}}\right] / C
$$

where $i=1,2$ and $C$ is the iron concentration in the sample (in $\mathrm{mM}),\left(1 / T_{i}\right)$ is the nuclear relaxation rate and the suffixes $\mathrm{s}$ and $\mathrm{d}$ stand for the sample and diamagnetic host (in this case of phosphate buffer saline at physiological $\mathrm{pH}$ ), respectively.

The relaxivity as a function of Larmor frequency, for S- and D-series SPIONs, in the absence and presence of a PC is reported in Fig. 6. From Fig. 6a, one can see that the longitudinal relaxivity of the SPIONs presents the typical behaviour of superparamagnetic contrast agents. In fact, the main mechanisms that induce nuclear longitudinal relaxation in the SPIONs are as follows: $:^{23,31}$ (a) for $\nu<1-5 \mathrm{MHz}$, the Neel relaxation of the particle magnetization, giving a correlation time related to the magnetic anisotropy barrier, and an associated reversal time, $\tau_{\mathrm{N}}$, that follows the Arrhenius law; (b) for $\nu>1-5 \mathrm{MHz}$, the Curie relaxation, which takes into account the sample magnetization through (the square of) the Langevin function weighted by the spectral density function $J^{\mathrm{F}}\left(\omega_{\mathrm{D}}\right)$, where $\omega_{\mathrm{D}}=1 / \tau_{\mathrm{D}}, \tau_{\mathrm{D}}$ being the self-diffusion correlation time of water. While mechanism (a) gives a flattening of $r_{1}(\nu)$ at frequencies $\nu<1-5 \mathrm{MHz}$, mechanism (b) is responsible for the maximum in $r_{1}(\nu)$ at higher frequencies $\nu>1-5 \mathrm{MHz}$.

Generally for SPIONs, the higher the transverse relaxivity, $r_{2}$, the better the contrast efficiency. Fig. $6 \mathrm{~b}$ plots $r_{2}$ as a function of the Larmor frequency for both the S- and D-series SPIONs. All samples demonstrated transverse relaxivities comparable with, or better than, Endorem ${ }^{\circledR}$. The S-plain and Endorem ${ }^{\circledR}$ presented the same relaxivity values, as expected, while the relaxivity value was smaller for the D-plain (which has a bigger hydrodynamic size). Furthermore, D-positive and S-negative SPIONs presented relaxivity values higher than that of Endorem ${ }^{\circledR}$.

Fig. 6c-f illustrate the relaxivity profiles of S- and D-series SPIONs before and after the formation of the PC. The longitudinal relaxivities, reported in Fig. $6 c$ and e, feature the typical behavior of superparamagnetic particles, even in the presence of the PC. 
To evaluate the effect of the PC on the $r_{2}$, the transverse relaxivities have been plotted in Fig. $6 \mathrm{~d}$ and $\mathrm{f}$ for the $\mathrm{S}$ - and $\mathrm{D}$ series SPIONs, respectively. The S-series SPIONs, the S-plain, Splain-PC, and Endorem ${ }^{\circledR}$, yielded almost the same relaxivity values (see Fig. 6d), indicating that the interaction between SPIONs and proteins does not substantially affect the relaxation times of the plain-dextran coated SPIONs.

On the other hand, the presence of the PC slightly increases the relaxivity of the negatively charged SPIONs and dramatically decreases the relaxivity in the case of the positively charged SPIONs. In other words, processes from protein adsorption to the carboxylate-amine coupling, i.e. NPs functionalized with $-\mathrm{COO}^{-}$ groups, do not affect the relaxivity of the SPIONs, while processes from protein adsorption to the amine-carboxylate coupling in the positively charged SPIONs, i.e. SPIONs functionalized with
$-\mathrm{NH}_{3}{ }^{+}$groups, decrease the relaxivity significantly despite the fact that protein absorption on the surface of positive SPIONs is significantly lower than negative SPIONs. The same behavior is seen for D-series SPIONs (see Fig. 6f), thus confirming the crucial role of the $-\mathrm{NH}_{3}{ }^{+}$group in decreasing $r_{2}$.

Additional insights can be gathered from the analysis of the longitudinal relaxivity data by resorting to the popular heuristic model by Roch and coworkers, ${ }^{35}$ which described the temperature dependence of $r_{1}$ as a linear combination of the limit cases of null and infinite anisotropy for an ensemble of SPIONs. The model depends critically on the minimum approach distance, $r_{\mathrm{a}}$, of the solvent protons to the magnetic core, and on the value of the volume saturation magnetization $M_{\mathrm{s}}$ of each particle, or, in other words, the single particle superparamagnetic moment. Assuming the value $D=2.3 \times 10^{-5} \mathrm{~cm}^{2} \mathrm{~s}^{-1}$ for the water
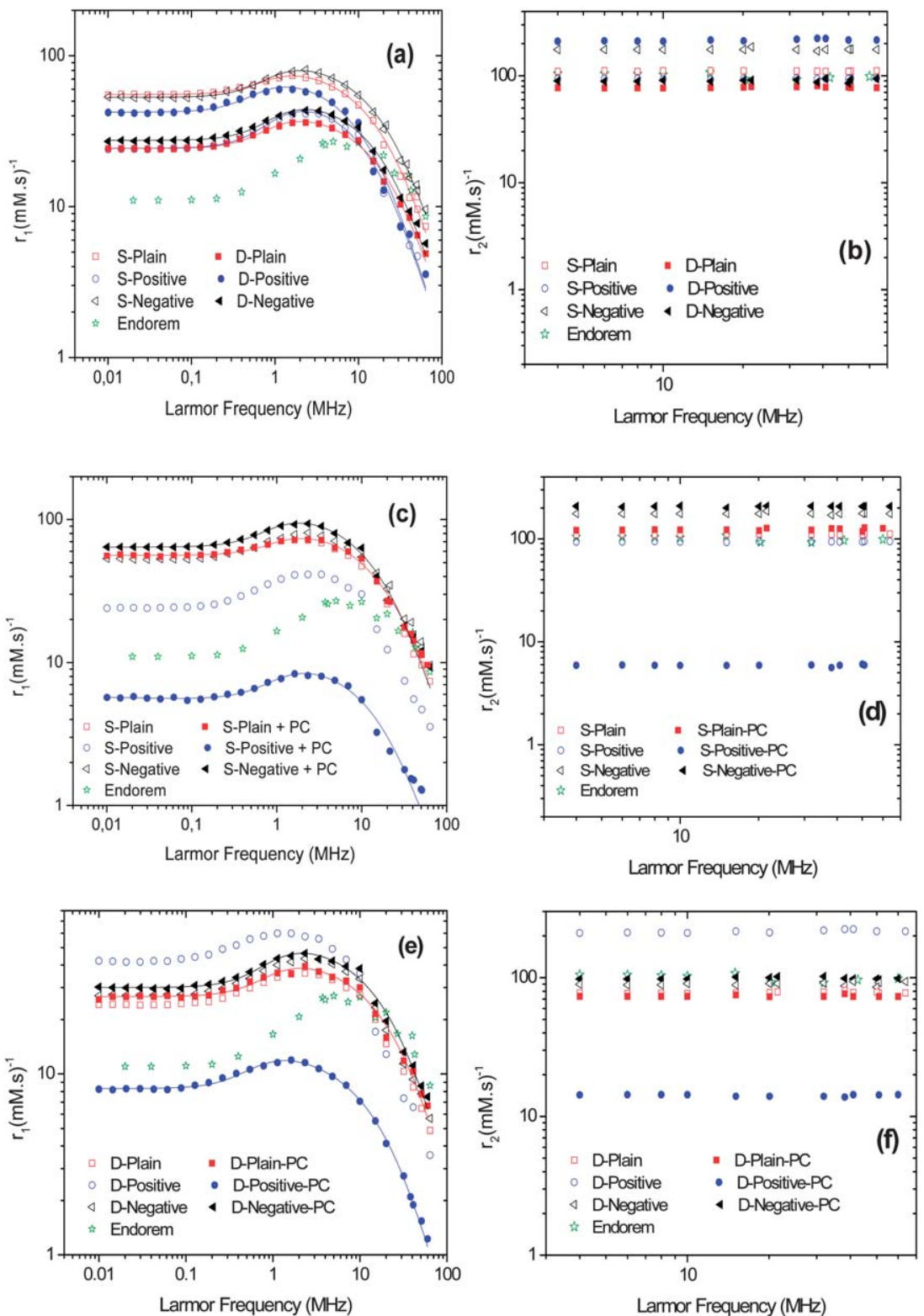

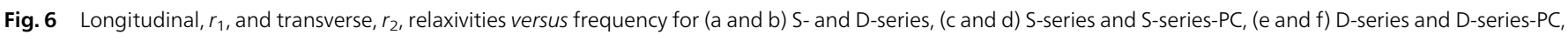
respectively. Solid lines are the best fit to the experimental data (see the text for fitting details). 
diffusion coefficient at room temperature and employing the $M_{\mathrm{s}}$ values obtained from magnetometry experiments, we extracted the minimum approach distance, $r_{\mathrm{a}}$, as a free fitting parameter. Fig. $6 \mathrm{a}, \mathrm{c}$ and e show the simulated curves that fit reasonably well to the experimental relaxometry profiles for all samples. The extracted $r_{\mathrm{a}}$ values are reported in Table 3 .

Solvent penetration can be evaluated by comparing the values of $r_{\mathrm{a}}$ to the nominal size of the particles; the values of $r_{\mathrm{a}}$ lie within a narrow range, between $7.7 \mathrm{~nm}$ and $9 \mathrm{~nm}$ for all cases. Since the nominal particle radius from TEM measurement was found to be between $7 \mathrm{~nm}$ and $8 \mathrm{~nm}$, the relaxometry results seem to suggest that the water molecules do not penetrate the organic shell, but they just graze the outer surface of the coating. Additionally, coating the particles with the protein corona does not alter the observed minimum approach distance, leading to the conclusion that the water molecules are able to penetrate into the adsorbed protein corona layer.

To further investigate the contrast efficiency of our samples, the $r_{2} / r_{1}$ ratio for the different sample series with and without PC has been plotted in Fig. 7. The $r_{2} / r_{1}$ ratio is an important indicator of the imaging contrast efficiency and usually, for $T_{2}$ (negative) contrast agents it is greater than 2. For positively charged SPIONs, $r_{2} / r_{1}$ is obviously altered when the SPIONs have their associated PC. This effect is not significant for the other samples.
In order to confirm the relaxometry findings, MRI experiments were performed to evaluate the SPIONs efficiency in MRI contrast enhancement in the presence of the PC. The experiments were performed for all samples, before and after incubation with serum proteins, at $8.5 \mathrm{MHz}$. Prior to the experiments, the iron concentration of all samples was carefully fixed to allow direct comparison of results. Fig. 8a shows an image of the SPION samples before any interaction with the proteins. Fig. $8 \mathrm{~b}$ and $\mathrm{c}$ present images of $\mathrm{S}$ - and D-series SPIONs, respectively, before and after the formation of the PCs. In all images, Endorem ${ }^{\circledR}$ is presented as a reference, and high resolution spin echo was used as the imaging sequence. From Fig. 8, it is apparent that (i) S-plain, S-plain-PC, and Endorem ${ }^{\circledR}$ present the same contrast, (ii) the contrast of the S-negative in the presence of proteins is enhanced slightly, (iii) in the case of S-positive SPIONs a significant decrease of the contrast is obvious in the presence of proteins, (iv) the presence of the PC does not affect the contrast of the D-plain SPIONs, while it increases slightly the contrast of the D-negative SPIONs, and decreases the contrast of the D-positive SPIONs considerably.

As described by Huang et al., MRI contrast comes from the difference in signal between water molecules present in different environments that are under the effect of magnetic nanoparticles. Because the interactions between water and the magnetic nanoparticles occur primarily on the surface of the
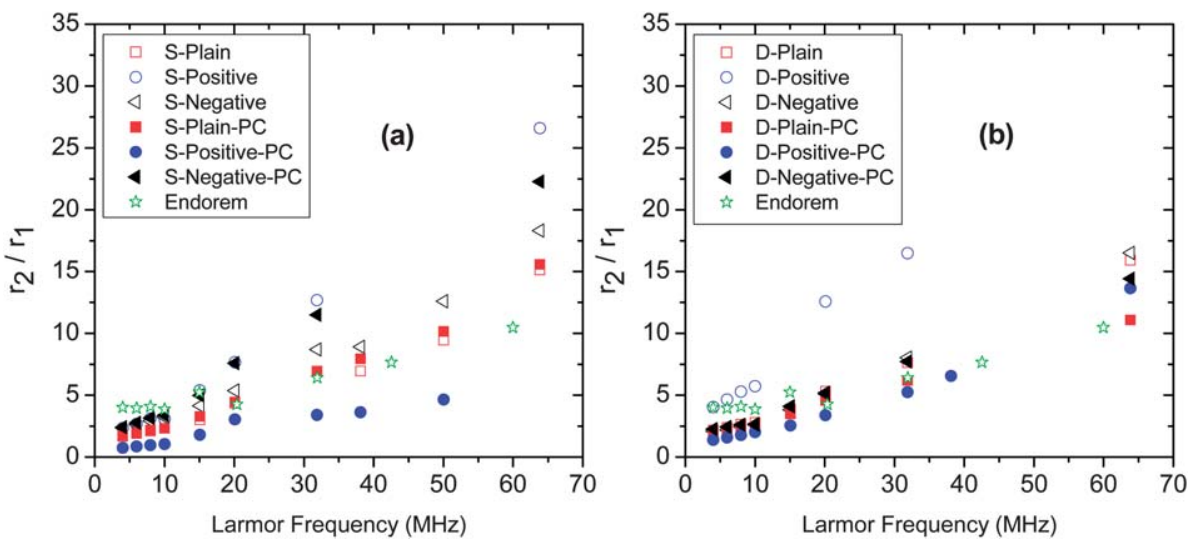

Fig. $7 r_{2} / r_{1}$ ratio for (a) S-series and (b) D-series samples in the absence and presence of the PC.
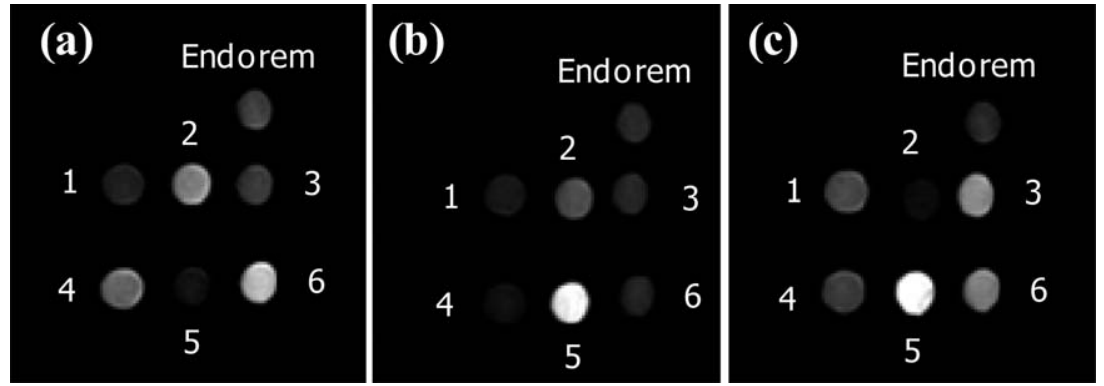

Fig. 8 MR images of SPION samples in vials at $\nu=8.5 \mathrm{MHz}$ using Artoscan by Esaote S.p.A. A high resolution spin echo sequence was employed and images are of (a) samples before incubation with protein; 1: S-negative, 2: S-positive, 3: S-plain, 4: D-negative, 5: D-positive, and 6: D-plain; (b) S-series with and without the associated proteins; 1: S-negative, 2: S-positive, 3: S-plain, 4: S-negative-PC, 5: S-positive-PC, and 6: S-plain-PC; (c) D-series with and without the associated proteins; 1: D-negative, 2: D-positive, 3: D-plain, 4: D-negative-PC, 5: D-positive-PC, 6: D-plain-PC. Imaging parameters are given in the text. 
nanoparticles, surface properties of magnetic nanoparticles play an important role in their magnetic properties and the efficiency for MRI contrast enhancement, ${ }^{32}$ and indeed it is reasonable to assume that the subsequent interaction with biomolecules in vivo would further modulate the contrast enhancement, as indicated in the work presented here. Noncovalent binding of contrast agents to HSA protein in vivo has been used to obtain contrast enhancement by slowing down the tumbling rate of the molecule. Indeed, the mechanism of the commercial agent Vasovist ${ }^{\circledR}$ (Gd-based CA) is based on this strategy. ${ }^{33}$ Coating affects the relaxation of water molecules via diffusion, hydration and hydrogen binding. ${ }^{32}$ Duan et $a .^{34}$ found that proton relaxivities depend on the surface hydrophilicity and coating thickness in addition to the particle size, as these affect the accessibility of water to the magnetic core and water exchange and diffusion between the bulk phase and the adjacent layer surrounding the particle surface.

The fact that the protein corona has the most dramatic effect on the positive SPIONs suggests an additional water structuring effect from the amine groups which requires further work to be elucidated.

\section{Conclusions}

To our knowledge, this is the first report of an effect of NPprotein interactions, and the formation of an adsorbed protein interface or corona, on the intrinsic magnetic properties and MRI contrast efficiency of SPIONs. In this report, the effects of the presence of a PC on the MRI contrast efficiency of two series of SPIONs with different hydrodynamic sizes (coating layer thicknesses) and surface charges (i.e. plain, negative and positive) were investigated. The PC was found to affect the magnetic properties and the MRI contrast efficiency of the SPIONs to different extents depending on their different PC compositions.

Physico-chemical characterization of the SPIONs was performed in the pristine state following incubation with a representative biological fluid (i.e. FBS). The protein corona layer affected the magnetic properties and the MRI contrast efficiency in a manner that was dependent on the specific characteristics of the dextran coating surrounding the SPIONs. Longitudinal relaxivities of the SPIONs demonstrated the typical behavior of superparamagnetic NPs, even following the association with proteins, and their transverse relaxivity values were comparable with or better than the (former) commercial product Endorem ${ }^{\circledR}$.

The singly coated (S-plain, S-plain-PC) SPIONs and Endorem ${ }^{\circledR}$ presented almost the same relaxivity values. The carboxylate-amine coupling in the negatively charged dextran coating of the SPIONs did not affect the relaxivity, while the amine-carboxylate coupling in the positively charged SPIONs decreased the relaxivity significantly. The composition of the PC on the surface of the SPIONs was affected by the surface chemistries of the SPIONs; there were very significantly lower protein amounts in the coronas of the positively charged SPIONs relative to the plain and especially the negatively charged SPIONs.
The in vitro MRI experiments confirmed the relaxometry findings regarding the impact of the PC on the contrast efficiency of SPIONs. The results suggest that such interactions should be taken into account prior to the development and optimization of multifunctional SPION systems for biomedical applications such as targeted molecular/cellular imaging and hyperthermia.

\section{Acknowledgements}

MM was funded via an ESF EpitopeMap Research Networking Programme Exchange grant to visit the Centre for BioNano Interactions, to carry out the protein corona study.

\section{References}

1 I. Lynch and K. A. Dawson, Protein-nanoparticle interactions, Nano Today, 2008, 3, 40-47.

2 M. P. Monopoli, D. Walczyk, A. Campbell, G. Elia, I. Lynch, F. Baldelli Bombelli, et al. Physical-Chemical Aspects of Protein Corona: Relevance to In Vitro and In Vivo Biological Impacts of Nanoparticles, J. Am. Chem. Soc., 2011, 133, 2525-2534.

3 D. Walczyk, F. B. Bombelli, M. P. Monopoli, I. Lynch and K. A. Dawson, What the Cell "Sees" in Bionanoscience, $J$. Am. Chem. Soc., 2010, 132, 5761-5768.

4 M. P. Monopoli, F. B. Bombelli and K. A. Dawson, Nanobiotechnology: nanoparticle coronas take shape, Nat. Nanotechnol., 2011, 6, 11-12.

5 M. Mahmoudi, I. Lynch, R. Ejtehadi, M. P. Monopoli, B. F. Baldelli and S. Laurent, Protein-Nanoparticle Interactions: Opportunities and Challenges, Chem. Rev., 2013, 5, 2570-2588.

6 I. Lynch, T. Cedervall, M. Lundqvist, C. Cabaleiro-Lago, S. Linse and K. A. Dawson, Adv. Colloid Interface Sci., 2007, 167, 134-135.

7 A. E. Nel, L. Mädler, D. Velegol, T. Xia, E. M. V. Hoek, P. Somasundaran, et al. Understanding biophysicochemical interactions at the nano-bio interface, Nat. Mater., 2009, 8, 543-557.

8 M. Mahmoudi, M. Hosseinkhani, S. Laurent, A. Simchi, H. Hosseinkhani, W. S. Journeay, et al. Magnetic Resonance Imaging Tracking of Stem Cells In Vivo Using Iron Oxide Nanoparticles as a Tool for the Advancement of Clinical Regenerative Medicine, Chem. Rev., 2011, 111, 253280.

9 M. Mahmoudi, I. Lynch, M. R. Ejtehadi, M. P. Monopoli, F. B. Bombelli and S. Laurent, Protein-nanoparticle interactions: possibilities and limitations, Chem. Rev., 2011, 111, 5610-5637.

10 M. Mahmoudi, K. Azadmanesh, M. A. Shokrgozar, W. S. Journeay and S. Laurent, Effect of nanoparticles on the cell life cycle, Chem. Rev., 2011, 111, 3407-3432.

11 A. Amirfazli, Nanomedicine: magnetic nanoparticles hit the target, Nat. Nanotechnol., 2007, 2, 467-468.

12 E. Amstad, J. Kohlbrecher, E. Muller, T. Schweizer, M. Textor and E. Reimhult, Triggered release from liposomes through 
magnetic actuation of iron oxide nanoparticle containing membranes, Nano Lett., 2011, 11, 1664-1670.

13 M. Arruebo, R. Fernández-Pacheco, M. R. Ibarra and J. Santamaría, Magnetic nanoparticles for drug delivery, Nano Today, 2007, 2, 22-32.

14 M. A. Dobrovolskaia and S. E. McNeil, Immunological properties of engineered nanomaterials, Nat. Nanotechnol., 2007, 2, 469-478.

15 S. Tong, S. Hou, Z. Zheng, J. Zhou and G. Bao, Coating optimization of superparamagnetic iron oxide nanoparticles for high $T_{2}$ relaxivity, Nano Lett., 2010, 10, 4607-4613.

16 A. Boni, M. Marinone, C. Innocenti, C. Sangregorio, M. Corti, A. Lascialfari, et al. Magnetic and relaxometric properties of Mn ferrites, J. Phys. D: Appl. Phys., 2008, 41, 134021.

17 M. F. Casula, P. Floris, C. Innocenti, A. Lascialfari, M. Marinone, M. Corti, et al. Magnetic resonance imaging contrast agents based on iron oxide superparamagnetic ferrofluids, Chem. Mater., 2010, 22, 1739-1748.

18 H. Amiri, R. Bustamante, A. Millan, N. J. Silva, R. Piol, L. Gabilondo, et al. Magnetic and relaxation properties of multifunctional polymer-based nanostructured bioferrofluids as MRI contrast agents, Magn. Reson. Med., 2011, 66, 1715-1721.

19 H. Amiri, M. Mahmoudi and A. Lascialfari, Superparamagnetic colloidal nanocrystal clusters coated with polyethylene glycol fumarate: a possible novel theranostic agent, Nanoscale, 2011, 3, 1022-1030.

20 M. Corti, A. Lascialfari, M. Marinone, A. Masotti, E. Micotti, F. Orsini, et al. Magnetic and relaxometric properties of polyethylenimine-coated superparamagnetic MRI contrast agents, J. Magn. Magn. Mater., 2008, 320, e316-e319.

21 M. Corti, A. Lascialfari, E. Micotti, A. Castellano, M. Donativi, A. Quarta, et al. Magnetic properties of novel superparamagnetic MRI contrast agents based on colloidal nanocrystals, J. Magn. Magn. Mater., 2008, 320, e320-e323.

22 M. Mahmoudi, S. Sant, B. Wang, S. Laurent and T. Sen, Superparamagnetic iron oxide nanoparticles (SPIONs): development, surface modification and applications in chemotherapy, Adv. Drug Delivery Rev., 2011, 63, 24-46.

23 S. Laurent, D. Forge, M. Port, A. Roch, C. Robic, L. Vander Elst, et al. Magnetic iron oxide nanoparticles: synthesis, stabilization, vectorization, physicochemical characterizations and biological applications, Chem. Rev., 2008, 108, 2064-2110.
24 N. Lee, Y. Choi, Y. Lee, M. Park, W. K. Moon, S. H. Choi, et al. Water-dispersible ferrimagnetic iron oxide nanotubes with extremely high $r(2)$ relaxivity for highly sensitive in vivo MRI of tumors, Nano Lett., 2012, 12, 3127-3131.

25 R. Weissleder, A. Moore, U. Mahmood, R. Bhorade, H. Benveniste, E. A. Chiocca, et al. In vivo magnetic resonance imaging of transgene expression, Nat. Med., 2000, 6, 351-354.

26 T. C. Usher and S. H. Wallis, Process of Making Carboxilated Dextran, US Pat., Number US6 703 499, 2004.

27 S. Martwiset, A. E. Koh and W. Chen, Nonfouling characteristics of dextran-containing surfaces, Langmuir, 2006, 22, 8192-8196.

28 M. R. Saboktakin, R. M. Tabatabaie, A. Maharramov and M. A. Ramazanov, A synthetic macromolecule as MRI detectable drug carriers: aminodextran-coated iron oxide nanoparticles, Carbohydr. Polym., 2010, 80, 695-698.

29 M. Mahmoudi, A. Simchi, A. S. Milani and P. Stroeve, Cell toxicity of superparamagnetic iron oxide nanoparticles, $J$. Colloid Interface Sci., 2009, 336, 510-518.

30 M. Mahmoudi, A. Simchi, M. Imani, A. S. Milani and P. Stroeve, Optimal design and characterization of superparamagnetic iron oxide nanoparticles coated with polyvinyl alcohol for targeted delivery and imaging, J. Phys. Chem. B, 2008, 112, 14470-14481.

31 A. Roch, R. N. Muller and P. Gillis, Theory of proton relaxation induced by superparamagnetic particles, $J$. Chem. Phys., 1999, 110, 5403-5411.

32 J. Huang, X. Zhong, L. Wang, L. Yang and H. Mao, Improving the magnetic resonance imaging contrast and detection methods with engineered magnetic nanoparticles, Theranostics, 2012, 2, 86-102.

33 E. J. Werner, A. Datta, C. J. Jocher and K. N. Raymond, Highrelaxivity MRI contrast agents: where coordination chemistry meets medical imaging, Angew. Chem., Int. Ed., 2008, 47, 8568-8580.

34 H. Duan, M. Kuang, X. Wang, Y. A. Wang, H. Mao and S. Nie, Reexamining the effects of particle size and surface chemistry on the magnetic properties of iron oxide nanocrystals: new insights into spin disorder and proton relaxivity, J. Phys. Chem. C, 2008, 112, 8127-8131.

35 A. Roch, R. N. Muller and P. Gillis, Theory of proton relaxation induced by superparamagnetic particles, $J$. Chem. Phys., 1999, 110, 5403-5411. 